\title{
Effect of pre-germination time of brown rice on serum cholesterol levels of hypercholesterolaemic rats.
}

\begin{abstract}
Background: Brown rice is unpolished rice with immeasurable benefits for human health. Brown rice (BR) and pre-germinated brown rice (PGBR) are known to contain various functional compounds such as gamma-oryzanol, dietary fibre and gamma-aminobutyric acid (GABA). In the present study, the experimental diets containing BR and PGBR (24, $48 \mathrm{~h}$ pregermination) were used to investigate the influence of pre-germination time of brown rice on blood cholesterol in Sprague-Dawley male rats. Result: Hypercholesterolaemia and elevation of LDL-cholesterol were successfully ameliorated by the experimental diets containing BR and PGBR (24 and $48 \mathrm{~h}$ pre-germination). As compared to the control sample, the pregermination time had a significant $(\mathrm{P}<0.05)$ effect on blood cholesterol of Sprague-Dawley male rats. It was also found that the significantly $(\mathrm{P}<0.05)$ better effect on lipid profile of hypercholesterolaemic rats was observed by prolonging the pre-germination time. As compared to non-germinated brown rice, the germinated brown rice showed the higher cardio-protective effect on hypercholesterolaemic Sprague-Dawley male rats. Conclusion: The present study suggests that the changes of blood cholesterol can be mainly modulated by using the PGBR rather than BR. The prolonging of pre-germination time led to an increase in the bioactive components, thereby providing a more efficient functional diet affecting the high blood cholesterol. This study suggests that PGBR can be used instead of BR and polished rice in the human diet.
\end{abstract}

Keyword: $\gamma$-amino butyric acid (GABA); $\gamma$-oryzanol; Brown rice; Dietary fibre; Hypercholesterolaemia; Pre-germination. 